\title{
A Noise Tolerant Watershed Transformation with Viscous Force for Seeded Image Segmentation
}

\author{
Di Yang, Stephen Gould, and Marcus Hutter \\ Research School of Computer Science, \\ The Australian National University
}

\begin{abstract}
The watershed transform was proposed as a novel method for image segmentation over 30 years ago. Today it is still used as an elementary step in many powerful segmentation procedures. The watershed transform constitutes one of the main concepts of mathematical morphology as an important region-based image segmentation approach. However, the original watershed transform is highly sensitive to noise and is incapable of detecting objects with broken edges. Consequently its adoption in domains where imaging is subject to high noise is limited. By incorporating a high-order energy term into the original watershed transform, we proposed the viscous force watershed transform, which is more immune to noise and able to detect objects with broken edges.
\end{abstract}

\section{Introduction}

Image segmentation for identification of homogeneous regions in an image has been the subject of considerable research activities over the last three decades. Here, segmentation refers to the process of partitioning a digital image into multiple contiguous regions (sets of pixels, also known as super-pixels). Such representations, over regions rather than individual pixels, may be more meaningful and easier to analyse. For example, image segmentation can be used as a pre-processing step for locate objects in images. More formally, image segmentation can be thought of as the process of assigning a label to every pixel in an image such that pixels with the same label share certain visual characteristics and belong to the same region. Boundaries between regions are defined whenever neighbouring pixels differ in their assigned labels.

Most image segmentation approaches can be divided into two classes, namely region-based and edge-based. One of the earliest prototypes for region-based segmentation is the Mumford-Shah functional [1,2], whose piecewise formulation is a spatially contiguous generalization of the Ising model [3]. Inspired by mean intensities employed in the Mumford-Shah functional, graph-based algorithms have been developed where globally optimal solutions (with respect to an energy function) can be obtained (e.g., graph-cuts [4] and watershed algorithms [5]). Other approaches, such as region-based level-sets (e.g., [1]), are similar but global optima cannot, in general, be guaranteed. 


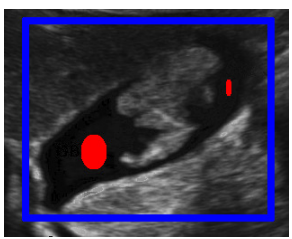

(a) Image \& seeds

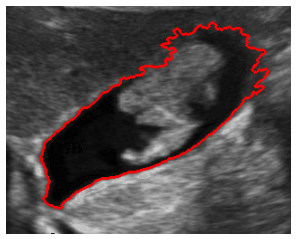

(e) Power watershed

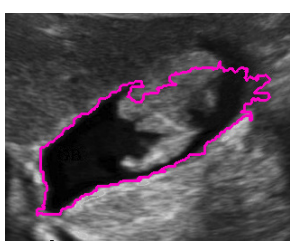

(b) Graph cuts

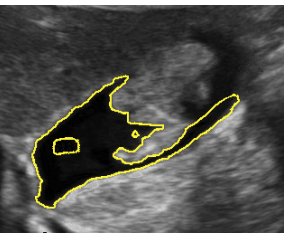

(f) RSF

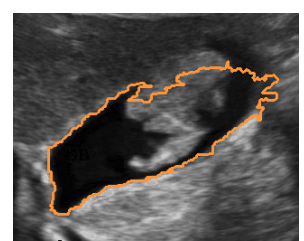

(c) Randon walker

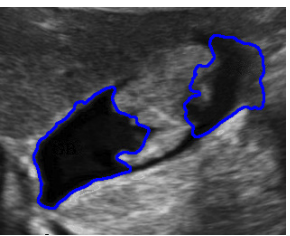

(g) DRLSE

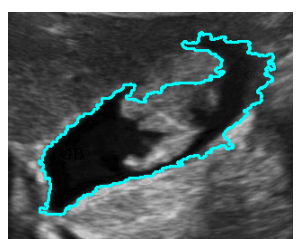

(d) Meyer flooding

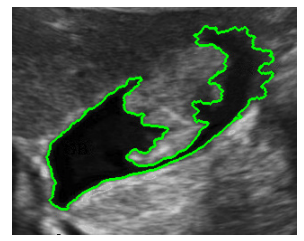

(h) Viscous watershed

Fig. 1: Ultrasonic image segmentation: (a) Gallbladder sludge ultrasonic image with predefined seeds (from http://www.ultrasound-images.com). Results produced by various algorithms: (b) Graph cuts; (c) Random walker [10]; (d) Meyer flooding [11]; (e) Power watershed [12]; (f) Region scalable level-sets (RSF) [2]; (g) Distance regularized level-sets evolution (DRLSE) [13]; (h) Our viscous watershed.

Edge-based approaches often adopt line integrals along proposed boundaries to score segmentations. The Snakes active contour model [6] is an early example of this approach. Here, curvature and length constraints are encoded as regularity terms into the integration of squared image gradients. Some variational variants [7] successfully incorporate gradient vector flow energy into the integral to enhance robustness. Level-set methods [8] define a powerful curve propagation scheme which has been proposed as replacement to the arc-length function [9] in active contour models for curve evolution. Unfortunately the quality of solutions from these approaches rely on difficult to tune parameters which are image specific.

Watershed image segmentation was originally proposed by Digable and Lantuejoul [5], and later improved by Beucher and Lantuejoul [14]. The method has been demonstrated as a powerful, non-parametric and fast technique for regionbased segmentation. In particular, watershed-based algorithms are widely used in image segmentation because of their efficiency and accuracy when applied to high-quality images. However, the watershed transformation suffers from poor robustness that results in significant fluctuations of the segmentation when contours are blurred or images are noisy. Moreover, the watershed transformation cannot find the outlines of objects with broken edges. Consequently, leaks and degeneracy may occur in results from watershed image segmentation (and indeed, other seeded image segmentation techniques). Figure 1, for example, highlights the failures of a number of segmentation algorithms on a low-quality image, whereas the performance of our algorithm is significantly better. 
In this paper, we propose a new method that can significantly improve the robustness of the watershed transformation by incorporating a high-order energy term (that we designate viscous force). Our viscous force watershed transformation has two main advantages over the original watershed transformation:

1. Our high-order energy term can dramatically enhance the robustness of the watershed transformation thereby making it more tolerant to noise and other forms of image corruption.

2. Our viscous force watershed transformation can extract the contour of objects with broken edges, which is not possible using the conventional watershed transformation.

Importantly, our method retains the key strengths of the original watershed transform - it is simple and fast. This makes our method of particular relevance to application domains requiring segmentation of low-quality images (e.g. medical ultrasonic images as shown in Figure 1).

\section{Background and Related Work}

The essential idea behind the watershed transformation can be understood from a geographic analogy: We consider a grey-scale image as a topographic relief where the intensity of a pixel is represented by the height of the relief. When a drop of water falls on a topographic relief, it moves downhill coming to rest within a local basin. By filling the topographic relief with water up to the point where water from different local basins meet we can identify so-called watershed lines. As a result, the landscape (or image) is partitioned into regions separated by the watershed lines.

Despite its simplicity, the watershed transform suffers from several problems. Primary amongst these is its high sensitivity to variations in the image gradient resulting in significant over-segmentation of the image. To solve this problem, marker-based approaches have been proposed within the watershed framework (e.g., optimal spanning forest [15] or Meyer's flooding [11]). Here markers (or seeds) may be interactively placed by users or found automatically using prior information to constrain the segmentation of the image into desired regions.

The most recent marker-based watershed image segmentation method is the power watershed method [12], which casts the watershed algorithm as an energy minimisation problem. As a consequence the method unifies various markerbased image segmentation algorithms such as graph-cuts, random walker and shortest path optimisation algorithms. This is achieved by replacing the objective in the traditional watershed method with a pairwise energy terms, which, to some extent, can partially improve robustness. However, without introducing high-order energy terms, the power watershed method is still unable handle low quality images or images corrupted by noise.

To address the problem of image corruption, Meyer [16,17] proposed the concept of viscous flooding. The idea of viscous flooding was inspired by morphological operations and geometrical constraints, and can be incorporated into 
the watershed segmentation algorithm in two different ways. The first, proposed by Meyer [16,17], is to simulate a viscous fluid in the watershed line construction via the opening morphological operation. Here the magnitude of viscous force only depends on the radius of the morphological operation rather than neighbourhood intensity changes in the image.

The second method for incorporating viscous flooding into the watershed transformation is via the topological relief regularisation [18]. The essence of this approach is still based on morphological opening. However, the aim is to regularise topographic relief directly with a morphological operation rather than simulating the viscous fluid flooding as in the first approach [16]. Unlike these approaches, our method directly connects neighbouring intensity changes to the magnitude of the viscous force.

Edge-based image segmentation algorithms tackle the problem from a different perspective. Many of these algorithms are based on the level-sets method [8], which evolves a contour to segment regions from a rough initial boundary. The most recent level-sets method is the distance regularised level set evolution algorithm (DRLSE) [13]. However, level-sets are not limited to edge-based algorithms. The approach can also be extended to region-based segmentation (e.g., [1]). The most recent level-sets approach belonging to this category is the region scalable fitting level set (RSF) algorithm [2]. In both of these approaches, however, level-sets methods can not guarantee the globally optimal result.

All of the methods described above may be considered as addressing energies or external forces comprised of only unary and pairwise terms. Watershed segmentations (Power watershed or Viscous watershed) only consider the low order terms (e.g., opened sets). However, recent literature has found that the addition of external force defined with higher order terms can help improve performance in a variety of tasks $[19,20]$. Although, level-set methods address image segmentation as energies and external forces comprised through pairwise terms, they failed to find the globally optimal result in most cases.

In this work, we propose a specific high-order term, which we call viscous force. By incorporating this term, we are able to address the drawbacks of previous approaches in dealing with noisy or corrupted images. Moreover, unlike the existing viscous watershed, our viscous force models neighbourhood intensity changes resulting in more precise and reliable segmentations.

\section{Viscous force into the watershed transformation}

We begin our exposition by reviewing the fundamentals of the watershed transformation [11]. We then present our high-order viscous force term and show how it improves the segmentation accuracy and robustness without sacrificing the performance even under low-quality imaging scenarios. We examine special cases of this algorithm in the context of noisy images and images with missing boundaries.

We will consider a finite grid of pixels. We embed the set of pixels within an undirected graph, where the graph $\mathrm{D}=(\mathrm{V}, \mathrm{E})$ consists of a set $\mathrm{V}=\{1,2, \cdots, n\}$ 
of vertices (or nodes) denoting the pixels and a set $\mathrm{E} \subseteq \mathrm{V} \times \mathrm{V}$ of pairs of vertices defining the connectivity structure, usually 4-connectivity or 8-connectivity. The set $\mathrm{E}$ consists of unordered pairs of nodes $(p, q)$ called an edge. In a weighted graph, a non-negative scalar (weight) is associated with each edge $(p, q) \in \mathrm{E}$ in the graph. We can now define a digital greyscale image as a 3 -tuple $\mathrm{D}=$ $(\mathrm{V}, \mathrm{E}, \mathrm{W}(\cdot))$, where $(\mathrm{V}, \mathrm{E})$ is a grid structured graph and $\mathrm{W}(\cdot)$ is an edge weight function $\mathrm{W}(p, q)$.

For many seeded image segmentation algorithms, the edge weights are determined by image intensity changes. One common format used by the graph-cuts and random walker segmentation algorithms is to set

$$
\mathrm{W}(p, q)=\exp \left(-\beta[I(p)-I(q)]^{2}\right),
$$

where $I(p)$ is the image intensity at node (pixel) $p$. However, a special topographic relief function $\mathrm{W}(\cdot)=\mathrm{T}_{\mathrm{f}}(\cdot)$ (mentioned later) will be employed in watershed segmentation.

Then, image segmentation proceeds to label each node (pixel) $p$ with a label from a fixed set $\mathrm{C}=\left\{c_{1}, c_{2}, \cdots, c_{n}\right\}$ according to its corresponding edge weights. We first label some of the nodes (pixels) with these labels and treat them as seeds. Then the seeded image segmentation for producing a segmentation is to solve the problem $[12,10,21]$

$$
\begin{array}{cl}
\underset{x}{\arg \min } & \sum_{(p, q) \in \mathrm{E}} \mathrm{W}(p, q) \llbracket x_{p}-x_{q} \rrbracket \\
\text { subject to } & x\left(c_{1}\right)=1, x\left(c_{2}\right)=2, \cdots, x\left(c_{n}\right)=n
\end{array}
$$

where $x_{p}$ denotes the unknown label of node (pixel) $p$. After establishing a function for the seeded image segmentation, there are some optimisation methods to search for the minima, for example region scalable level set (RSF) [2], graph cuts [4] and watershed algorithms [16].

As demonstrated in Figure 1, the above seeded image segmentation algorithms, associated with different optimisation methods, are unable to produce satisfactory segmentation results under low quality imaging. In other words, no mater what kind of optimisation method is employed by the seeded image segmentation, leaks and degeneracy frequently occur in the results under low quality imaging. That means the problem lies in the objective function rather than optimisation method. Thus, in this paper, we will incorporate a viscous force into the weight function, in order to produce a better weighted graph for segmentation.

\subsection{Review of watershed transformation}

We now describe how watershed segmentation simulates the fluid flooding and finds the watershed lines based on the established topographic relief.

Let the topographic relief $f(\cdot) \rightarrow[0,255]$ have minima $\left\{m_{k}\right\}_{k \in \mathrm{K}}$, shown in Figure 2 (a), for some index set $\mathrm{K} \subseteq \mathrm{V}$. The catchment basin $\mathrm{CB}\left(m_{i}\right)$ of a 


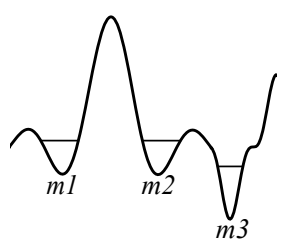

(a)

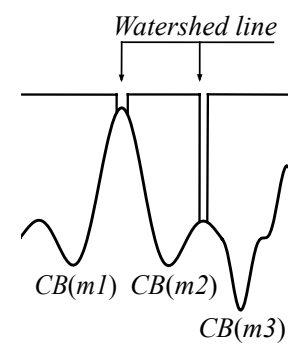

(b)

Fig. 2: Diagrams of minima, catchment basin and watershed line. From left to right: (a) Local minima of relief; (b) Yielded catchment basins and corresponding watershed lines.

minimum $m_{i}$ is defined as the set of nodes (pixels) $p \in \mathrm{V} \backslash \mathrm{K}$, which are topographically closer to $m_{k}$ with topographic distance $T_{f}\left(p, m_{k}\right)$ than to any other regional minimum $m_{l}$ with $T_{f}\left(p, m_{l}\right)$ (see Figure $2(\mathrm{~b})$ ):

$$
\mathrm{CB}\left(m_{k}\right)=\left\{p \in \mathrm{V}, k \in \mathrm{K} \mid \forall l \in \mathrm{K} \backslash\{k\}: f\left(m_{k}\right)+T_{f}\left(p, m_{k}\right)<f\left(m_{l}\right)+T_{f}\left(p, m_{l}\right)\right\} .
$$

In an infinite graph, the watershed line of $f$ is the set of pixels that specify the boundaries between regions. Formally the watershed line is defined as the set of pixels that does not belong to any catchment basin (see Figure 2 (b)):

$$
\operatorname{Wshed}(f)=\mathrm{V} \backslash\left[\bigcup_{k \in \mathrm{K}} \mathrm{CB}\left(m_{k}\right)\right] .
$$

Let Wshed denote the label for the watershed line - Wshed $\notin \mathrm{K}$. The watershed transform of $f$ is a mapping $\lambda: \mathrm{V} \rightarrow \mathrm{K} \cup\{$ Wshed\}, such that $\lambda(p)=k$ if $p \in \mathrm{CB}\left(m_{k}\right)$, and $\lambda(p)=\mathrm{W}$ if $p \in \mathrm{Wshed}(f)$. So the watershed transform of $f$ assigns labels to the points of $\mathrm{V}$, such that different catchment basins are uniquely labelled, and a special label $\mathrm{W}$ is assigned to all points on the watershed line.

Initially, we assume that the image $I$ is lower complete; that means each pixel which is not in a minimum has a neighbour with a lower grey value. A plateau situation will be discussed later. The gradient magnitude $\|\nabla I(p)\|$ of $I$ at a pixel $p$ could be used to describe the topographic distance. But, in practice, the lower slope $L S(p)$ is actually calculated as the topographic distance due to computational efficiency. It is defined as the maximal slope linking $p$ to any of its neighbour of lower altitudes. Formally,

$$
L S(p)=\max _{q \in N_{G}(p)}\left(\frac{I(p)-I(q)}{d(p, q)}\right)
$$

where $N_{G}(p)$ is the set of neighbours of the node (pixel) $p$ of the grid $\mathrm{D}=(\mathrm{V}, \mathrm{E})$, and $d(p, q)$ is the Manhattan distance between nodes (pixels) $p$ and $q$. Then, the 


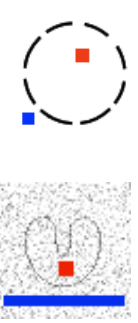

(a)
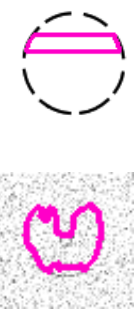

(b)

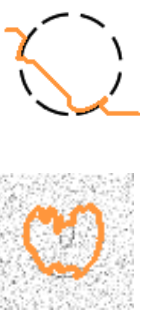

(c)
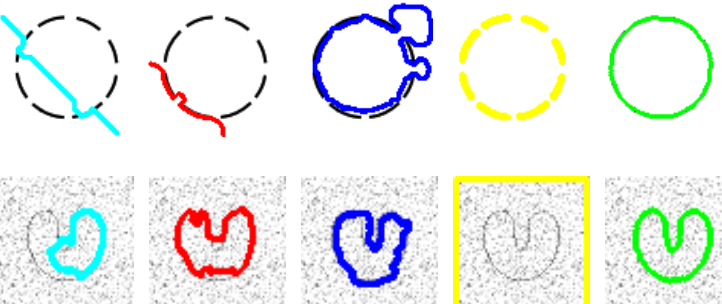

(e)

(d)

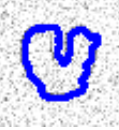

(f)

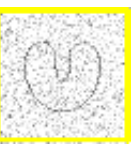

(g)

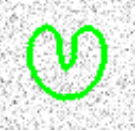

(h)

Fig. 3: Illustration of segmentations using predefined seeds in synthetic broken boundary and noise scenarios. (a) Image with seeds; (b - h) Segmentation results are yielded by Graph cuts, Random walker, Meyer flooding, Power watershed, DRLSE, RSF, and viscous force watershed, respectively. See text for details.

topographic distance from $p$ to a neighbouring node $q$ is defined as

$$
T_{f}\left(\nu_{p}, \nu_{q}\right)= \begin{cases}L S(p) \cdot d(p, q), & \text { if } I(p)>I(q) \\ L S(q) \cdot d(p, q), & \text { if } I(p)<I(q) \\ \frac{1}{2}(L S(p)+L S(q)) \cdot d(p, q), & \text { if } I(p)=I(q) .\end{cases}
$$

The topographical distance along a path $\pi=\left(p_{0}, \ldots, p_{l}\right)$ between $p_{0}=p$ and $p_{l}=q$ in $\mathrm{V}$ is defined by:

$$
T_{f}^{\pi}(p, q)=\sum_{i=0}^{l-1} T_{f}\left(p_{i}, p_{i+1}\right) .
$$

If an image is not lower complete the topographical distance between the interior pixels of a plateau will be identically zero. Thus, the Manhattan distance to the boundary of the plateau is usually computed instead of the topographic distance in this situation.

\subsection{Watershed transformation with viscous force}

Let $\mathrm{D}=\left(\mathrm{V}, \mathrm{E}, T_{f}\right)$ be a greyscale image. For any pair of vertices $p, q \in \mathrm{V}$, consider all paths from $p_{0}=p$ to $p_{l}=q$, whose vertices belong to the path $\pi=\left(p_{0}, p_{2}, \ldots, p_{l}\right)$ for which $\pi \subset \mathrm{V}$.

Let $T_{f}^{\pi \star}(p, q)$ be the minimum-weight path among them. If $p_{k}$ is on the shortest path $T_{f}^{\pi \star}(p, q)$, then we can break this path into two sub-paths: one from $p$ to $p_{k}$ as $T_{f}^{\pi}\left(p, p_{k}\right)$, the other from $p_{k}$ to $q$ like $T_{f}^{\pi}\left(p_{k}, q\right)$. Then, each vertices $p_{i}$ on the shortest path $\pi^{\star}$ would satisfy the following properties:

$$
T_{f}^{\pi \star}(p, q)= \begin{cases}T_{f}(p, q), & \text { if }(p, q) \in \mathrm{E} \\ \underset{p_{k} \in D,\left(p_{k}, q\right) \in E}{\arg \min }\left[T_{f}^{\pi \star}\left(p, p_{k}\right)+T_{f}^{\pi \star}\left(p_{k}, q\right)\right] & \text { otherwise. }\end{cases}
$$

However, Equation 8 only considers vertices on the path and ignores other vertices in the neighbourhood. This property yields a weakness in the watershed 
transform, because "fluid" may leak out from broken edge or be interfered by noisy pixels (see Figure 3). Thus, we introduce a high-order term to the topographic distance calculation, in order to improve robustness of the watershed algorithm.

Intuitively, the idea of our high-order term can be motivated by the averaging the cost of all paths in the neighbourhood of a given path rather than the cost of the path itself. We expect the average path to be more robust to local topographic variations (e.g. broken edges and noise). This energy term presents as follows

$$
\hat{T}_{f}^{\pi}(p, q)=\sum_{i=0}^{l-1}\left\{T_{f}\left(p_{i}, p_{i+1}\right)+\frac{1}{2}\left[\operatorname{VF}\left(p_{i}\right)+\operatorname{VF}\left(p_{i+1}\right)\right]\right\} .
$$

where $\operatorname{VF}(p)$ is the energy function associated with $p$, which calculates the averaging topographic distance from point $p$ on the path to the other points in the neighbourhood. We define $\operatorname{VF}(p)$ :

$$
\mathrm{VF}(p)=\frac{1}{C} \ln \left\{1+\sum_{q \in N_{G}(p)}\left[1-e^{-C \cdot T_{f}(p, q)}\right]\right\},
$$

where $C$ is a positive constant, which controls the magnitude of the viscous force per pixel. Smaller values of $C$ produce a larger viscous force. In the other words, the viscous force effects vanish for large $C$. In our experiments, we found that setting $C=0.005$ gives satisfactory results over a large range of images.

The size of the local neighbourhood $N_{G}(p)$ also affects the strength of the viscous force. When the neighbourhood is too large, the total viscous force may make fluid too thick to detect details of the topographic relief without a proper $C$, and our algorithm tends to over-smooth the image. Additionally, the computational cost of the viscous force depends on the size of this neighbouring set. We found that setting the neighborhood to $3 \times 3$ or $5 \times 5$ usually results in good performance. Then, equation 9 can be efficiently solved by the dynamic program, and Moore-Bellman-Ford algorithm is employed in this paper.

As shown in Figure 3(top row), the energy term assigns a high penalty value to the weighted path going through the window to make it expensive and stop the fluid from leaking out. Other seeded image segmentation approaches treat the broken edges as a plateau and give some unreasonable results.

In Figure 3(bottom row), the viscous force will result in a constant topographical path cost over local regions in noisy scenarios, which can neutralise the impact of noisy pixels. Other seeded image segmentation algorithms are affected by noisy pixels critically, so most of them cannot locate the boundaries of the structure or capture its shape.

After introducing the viscous force term into watershed transformation, we generate a new viscous force watershed transformation in equation 9 and 10 . We can also incorporate our weight function, incorporating the viscous force, into other seeded image segmentation approaches via the generic framework described above (i.e. equation 2). 

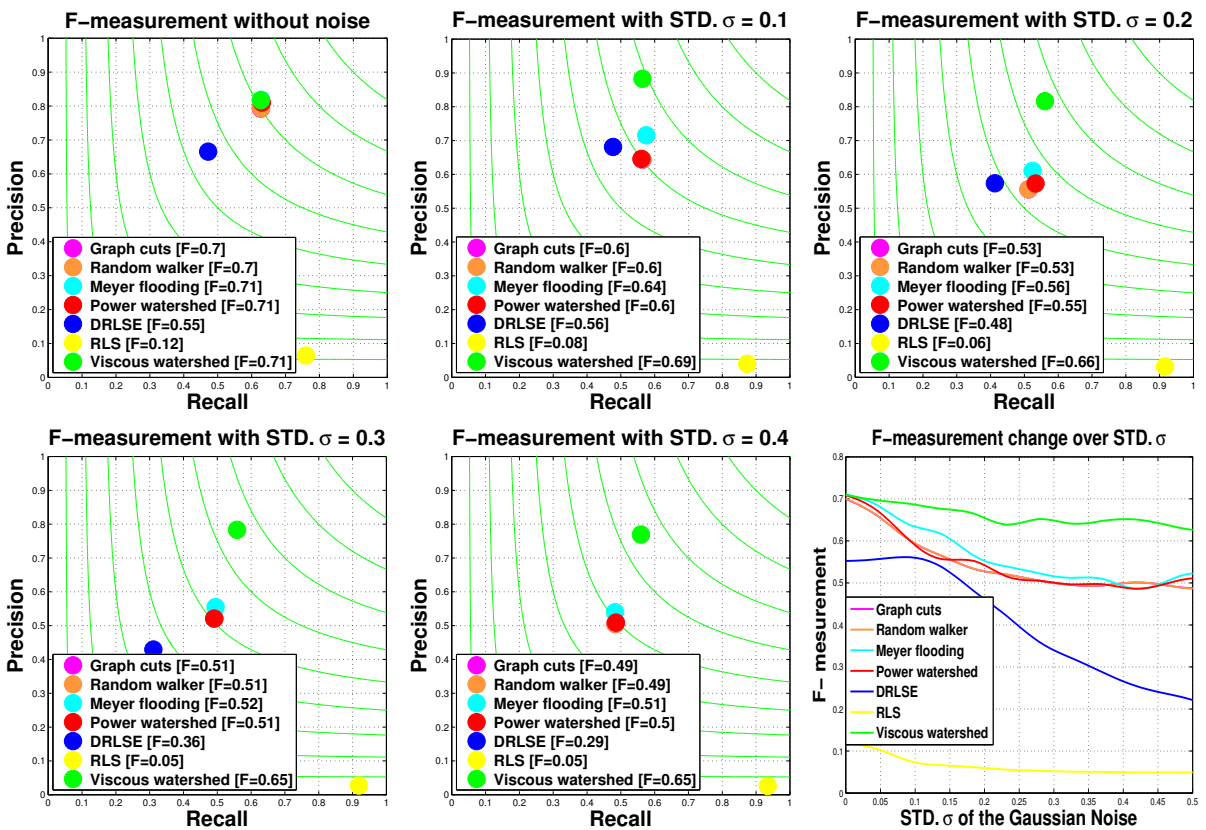

Fig. 4: Illustration of $\mathrm{F}$-measurement drawing in $P R$-chart under the different magnitude of gaussian noise. Top row: F-measurement on original images, and F-measurement on images corrupted by gaussian noise with standard deviations, $\sigma=0.1, \sigma=0.2$. Bottom row: F-measurement on images corrupted by Gaussian noise with standard deviations, $\sigma=0.3, \sigma=0.4$, and changes of F-measurements over different levels of noise. Measurement software is provided by BSDS500 dataset.

\section{Experimental results}

We evaluate the performance of our proposed viscous force watershed transformation against other seeded watershed image segmentation algorithms and active contour models - specifically graph cuts, random walker, Meyer flooding, power watersheds, DRLSE, and RSF. Our experiments use the 50 image GrabCut dataset [23]. This dataset was first proposed for evaluating interactive image segmentation algorithms. It provides foreground segmentation masks which we use to generate the seeds by applying significant erosion.

Our viscous force watershed is targeted at low-quality image segmentation, (see in Figure 1). To evaluate performance in this regime we corrupt the images in the GrabCut dataset by adding zero-mean gaussian noise $\left(\sigma \in\left[\begin{array}{ll}0.1 & 0.4\end{array}\right]\right)$ to each pixel. Quantitative evaluation is then performed using the precision-recall chart and F-measurement metrics developed by [24] for boundary quality evaluation. We also report ground truth covering and variation of information metric (VI) for region covering evaluation [25, 24]. 


\begin{tabular}{|c|c|c||c|c||c|c||c|c||c|c|}
\hline & \multicolumn{2}{|c|}{$\sigma=0$} & \multicolumn{2}{c|}{$\sigma=0.1$} & \multicolumn{2}{c||}{$\sigma=0.2$} & \multicolumn{2}{c||}{$\sigma=0.3$} & \multicolumn{2}{c|}{$\sigma=0.4$} \\
\cline { 2 - 11 } & MedF & BestF & MedF & BestF & MedF & BestF & MedF & BestF & MedF & BestF \\
\hline \hline Graph cuts & 0.700 & 0.792 & 0.659 & 0.727 & 0.564 & 0.592 & 0.520 & 0.537 & 0.496 & 0.511 \\
\hline Random walker & 0.701 & 0.793 & 0.660 & 0.728 & 0.564 & 0.592 & 0.520 & 0.537 & 0.496 & 0.512 \\
\hline Meyer flooding & $\mathbf{0 . 7 1 1}$ & $\mathbf{0 . 8 1 9}$ & 0.684 & 0.781 & 0.616 & 0.679 & 0.539 & 0.580 & 0.512 & 0.549 \\
\hline Power watershed & 0.709 & 0.810 & 0.671 & 0.743 & 0.556 & 0.586 & 0.515 & 0.534 & 0.496 & 0.510 \\
\hline DRLSE & 0.552 & 0.666 & 0.557 & 0.673 & 0.537 & 0.647 & 0.422 & 0.503 & 0.322 & 0.383 \\
\hline RSF & 0.119 & 0.165 & 0.104 & 0.179 & 0.066 & 0.112 & 0.056 & 0.091 & 0.051 & 0.093 \\
\hline Viscous watershed & 0.710 & 0.817 & $\mathbf{0 . 6 9 7}$ & $\mathbf{0 . 8 0 1}$ & $\mathbf{0 . 6 7 6}$ & $\mathbf{0 . 8 4 5}$ & $\mathbf{0 . 6 3 9}$ & $\mathbf{0 . 7 5 4}$ & $\mathbf{0 . 6 4 1}$ & $\mathbf{0 . 7 5 7}$ \\
\hline \hline$\Delta^{\dagger}$ & $\mathbf{- 0 . 0 0 1}$ & $\mathbf{- 0 . 0 0 2}$ & $\mathbf{0 . 0 1 3}$ & $\mathbf{0 . 0 2 0}$ & $\mathbf{0 . 0 6 0}$ & $\mathbf{0 . 1 6 6}$ & $\mathbf{0 . 1 0 0}$ & $\mathbf{0 . 1 7 4}$ & $\mathbf{0 . 1 2 9}$ & $\mathbf{0 . 2 0 8}$ \\
\hline
\end{tabular}

Table 1: F-measurement for boundary detection computed between the segmentations yielded by seven algorithms and ground truth images in GrabCut data set. $\dagger: \Delta$ shows difference in $\mathrm{F}$-measurement between our algorithm and the best algorithm among the remaining six.

\subsection{Boundary quality}

The F-measurement is particularly meaningful in the context of boundary maps. It is reasonable to characterise higher level processing in terms of how true a signal is required for $R$ (recall) to be successful, and how much noise can be tolerated $P$ (precision). Then a balanced F-measurement can be calculated from these quantities.

Table 1 shows the quantitative results for the seven algorithms being tested under different Gaussian noise scenarios. We report two quantities. The first (MedF) reports the median F-measurement across the 50-image dataset, and the second (BestF) reports the best F-measurement over all images in the dataset. These metrics (BestF and MedF) range from 0 to 1 corresponding to bad and good matches, respectively.

Some qualitative results are shown in Figure 4. As these images and the quantitative results show, segmentations produced by our viscous watershed on original (noise free) images are as good as results produced by the other seeded image segmentation algorithms. The difference of quantitative measurements is marginal in the noise-free case, and the seeded image segmentation approaches all perform much better than DRLSE and RSF. However, when segmenting in the presence of Gaussian noise, our viscous force watershed method outperforms the other six algorithms.

Additionally, Figure 4 shows that the F-measurement metric of our viscous force watershed method drop much slower than the other algorithms as the magnitude of noise increases. In other words, viscous force is more robust to noise than the other seeded image segmentation algorithms.

\subsection{Region quality}

The Variation of Information metric (VI) was introduced for the purpose of label comparison [25,24]. It measures the distance between two segmentations in terms of their average conditional entropies given by:

$$
\operatorname{VI}\left(S, S^{\prime}\right)=H(S)+H\left(S^{\prime}\right)-2 I\left(S, S^{\prime}\right),
$$



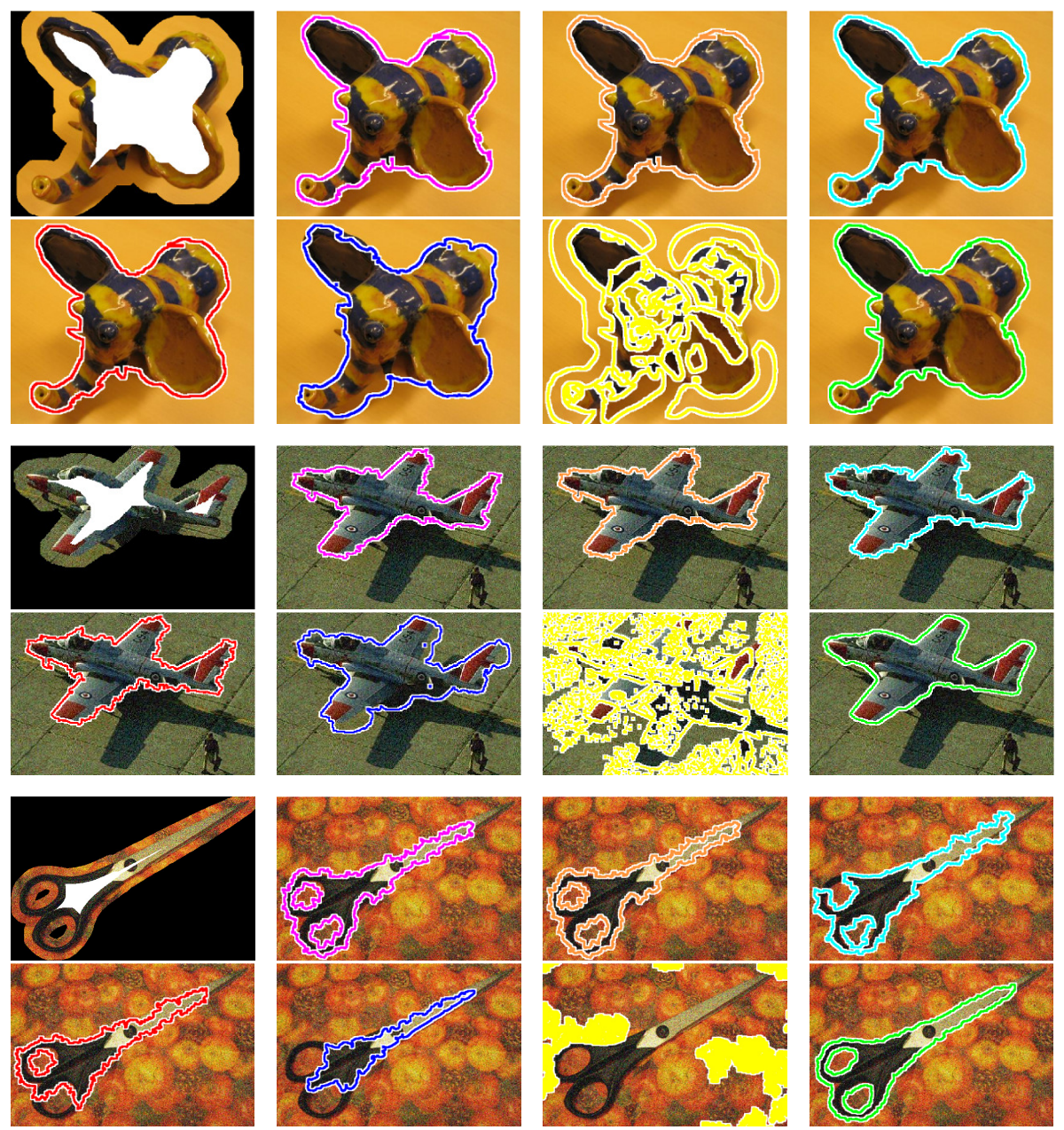

Fig. 5: Example segmentations on the GrabCut dataset images under different magnitudes of noise. For each image, top row: image with seeds, segmentations yielded by graph cuts, random walker, and Meyer flooding, bottom row: segmentations produced by power watershed, DRLSE, RSF and viscous watershed. From top to bottom, the ceramic elephant image is the original one without noise corruption, the fighter jet image is corrupted by a Gaussian noise with $\sigma=0.1$, and the scissors image is corrupted by a Gaussian noise with $\sigma=0.3$.

where $H$ and $I$ are the entropies and mutual information between two segmentations $S$ and $S^{\prime}$, respectively. Smaller VI means better segmentation.

Our second evaluation metric for region segmentation quality is ground truth covering defined as

$$
\mathcal{O}\left(R, R^{\prime}\right)=\frac{\left|R \cap R^{\prime}\right|}{\left|R \cup R^{\prime}\right|}, \quad C\left(S^{\prime} \rightarrow S\right)=\frac{1}{N} \sum_{R \in S}|R| \cdot \max _{R^{\prime} \in S^{\prime}} \mathcal{O}\left(R, R^{\prime}\right),
$$




\begin{tabular}{|c|c|c||c|c||c|c||c|c||c|c|}
\hline & \multicolumn{2}{|c||}{$\sigma=0$} & \multicolumn{2}{c|}{$\sigma=0.1$} & \multicolumn{2}{c||}{$\sigma=0.2$} & \multicolumn{2}{c||}{$\sigma=0.3$} & \multicolumn{2}{c|}{$\sigma=0.4$} \\
\cline { 2 - 13 } & Cover & VI & Cover & VI & Cover & VI & Cover & VI & Cover & VI \\
\hline Graph cuts & 0.971 & 0.200 & 0.954 & 0.295 & 0.939 & 0.373 & 0.937 & 0.386 & 0.937 & 0.384 \\
\hline Random walker & 0.971 & 0.199 & 0.954 & 0.295 & 0.939 & 0.373 & 0.937 & 0.386 & 0.934 & 0.384 \\
\hline Meyer flooding & $\mathbf{0 . 9 7 2}$ & 0.196 & 0.959 & 0.269 & 0.942 & 0.356 & 0.939 & 0.372 & 0.940 & 0.367 \\
\hline Power watershed & $\mathbf{0 . 9 7 2}$ & $\mathbf{0 . 1 9 1}$ & 0.951 & 0.299 & 0.940 & 0.371 & 0.937 & 0.386 & 0.938 & 0.382 \\
\hline DRLSE & 0.949 & 0.308 & 0.952 & 0.290 & 0.930 & 0.351 & 0.917 & 0.38 & 0.921 & 0.372 \\
\hline RSF & 0.479 & 1.606 & 0.500 & 1.574 & 0.501 & 1.550 & 0.496 & 1.561 & 0.492 & 1.570 \\
\hline Viscous watershed & $\mathbf{0 . 9 7 2}$ & 0.199 & $\mathbf{0 . 9 7 0}$ & $\mathbf{0 . 2 1 1}$ & $\mathbf{0 . 9 6 1}$ & $\mathbf{0 . 2 5 7}$ & $\mathbf{0 . 9 5 8}$ & $\mathbf{0 . 2 7 8}$ & $\mathbf{0 . 9 6 0}$ & $\mathbf{0 . 2 6 5}$ \\
\hline
\end{tabular}

Table 2: Region evaluation ground truth covering and Variation Information (VI), computed between the segmentations yielded by seven algorithms and ground truth images in GrabCut dataset.

\begin{tabular}{|l|l|l|l|l|l|l|l|}
\hline Algorithm & Graph cuts & $\begin{array}{l}\text { Random } \\
\text { walker }\end{array}$ & $\begin{array}{l}\text { Meyer } \\
\text { flooding }\end{array}$ & $\begin{array}{l}\text { Power wa- } \\
\text { tershed }\end{array}$ & DRLSE & RSF & $\begin{array}{l}\text { Viscous } \\
\text { watershed }\end{array}$ \\
\hline \hline exec. time & $247.4 \mathrm{~ms}$ & $2.6 \mathrm{~ms}$ & $6.8 \mathrm{~ms}$ & $353.8 \mathrm{~ms}$ & $32.9 \mathrm{~s}$ & $4.87 \mathrm{~s}$ & $56.27 \mathrm{~ms}$ \\
\hline
\end{tabular}

Table 3: Average executing time comparison among all these algorithms running on a 2.66GHz Intel Core2 Quad CPU Q9700 platform with Ubuntu 11.10.

where $R$ and $R^{\prime}$ are the overlapped regions, the covering index $C$ of the ground truth $S$ by a segmentation $S^{\prime}$ respect to $\mathcal{O}(\cdot)$, and, $N$ denotes the total number of pixels in the image.

The average over all 50 images of variation information and ground truth covering are shown in Table 2. According to these metrics the viscous force watershed performs better than all the other algorithms under noisy scenariosits segmentation has the smallest value of Variation Information and the highest measurements in ground truth covering.

Last, we compare the running time of these different seeded image segmentation algorithms (see Table 3). Our method along with the other graph-based algorithms are much faster than the level sets approaches. Although, our method is slightly slower than the random walker and Meyer's flooding, it is still fast enough for many real-time applications.

\subsection{Precision and Robustness}

One may consider using a pre-filter to smooth the images before applying the segmentation algorithms as an alternative to our viscous force watershed method. However, as shown in Table 4, per-filtering may improve the segmenting performance slightly, but they are still not as good as our algorithm. We believe that this is because the pre-noise filter decreases noise magnitude at the cost of losing some high-frequency information. As such, precisely localizing boundaries and edges is compromised.

The affect of viscous force is different from pre-filtering. The viscous force watershed is designed to find an averaging weighted path over all paths around a certain minimum path in a neighbourhood. Thus, unlike the pre-filtering process, our method can not only enhance segmentation robustness, but can also preserve high-frequency information (precision) instead of filtering it along with the noise. 


\begin{tabular}{|l|l|l|l|l|l|l|l|}
\hline$\sigma=\mathbf{0 . 2}$ & Graph C. & Random W. & Meyer F. & Power W. & DRLSE & RSF & Viscous W. \\
\hline MedF & 0.600 & 0.600 & 0.628 & 0.587 & 0.569 & 0.067 & $\mathbf{0 . 6 7 6}$ \\
\hline BestF & 0.604 & 0.604 & 0.630 & 0.598 & 0.665 & 0.1567 & $\mathbf{0 . 8 4 5}$ \\
\hline \hline cover. & 0.951 & 0.951 & 0.955 & 0.950 & 0.949 & 0.488 & $\mathbf{0 . 9 6 1}$ \\
\hline VI & 0.309 & 0.309 & 0.286 & 0.313 & 0.296 & 1.587 & $\mathbf{0 . 2 5 7}$ \\
\hline
\end{tabular}

Table 4: Performance evaluation on the pre-filtered images. Original images are corrupted by a Gaussian noise $(\sigma=0.2)$, A Gaussian filter $(\operatorname{size}=15 \times 15, \sigma=0.5)$

\section{Discussion and Conclusion}

In this paper we developed a high-order external energy term (viscous force) for image segmentation algorithms based on watershed. This energy term significantly improves the robustness of the watershed transformation. Specifically, our modification renders the watershed tolerant to noise and capable of segmenting object with broken edges.

Watershed-based algorithms have found many different applications in the computer vision field that go beyond image segmentation, such as stereo disparity map, video super-resolution and dynamic object detection [26-28]. By employing our viscous force formulation to improve robustness, the watershed transform may find even more applications within computer vision.

Our work, together with recent methods that unify the watershed transformation and graph-based segmentation techniques, suggest exciting future research directions. Most promising of these, perhaps, is the generalisation of our highorder viscous force approach to other seeded image segmentation algorithms including graph-cut and random walker via a unifying framework.

Acknowledgement: This work was kindly supported by ControlExpert GmbH, Langenfeld (Germany).

\section{References}

1. Chan, T., Vese, L.: Active contours without edges. IEEE Trans. on Image Processing 10 (2001) $266-277$

2. Li, C., Kao, C.Y., Gore, J., Ding, Z.: Minimization of region-scalable fitting energy for image segmentation. IEEE Trans. on Image Processing 17 (2008) 1940 -1949

3. Binder, K. Ising model. In: Hazewinkel, Michiel, Encyclopedia of Mathematics. Springer (2001)

4. Boykov, Y.Y., Jolly, M.P.: Interactive graph cuts for optimal boundary \& region segmentation of objects in N-D images. In: ICCV. (2001) 105-112

5. Digabel, H., Lantuejoul, C.: Iterative algorithms. In: European Symposium on Quantitative Analysis of Microstructures in Materials Sciences, Biology and Medicinen, Caen, France. (1977) 85-99

6. Kass, M., Witkin, A., Terzopoulos, D.: Snakes: Active contour models. IJCV 1 (1988) 321-331

7. Xu, C., Prince, J.L.: Snakes, shapes, and gradient vector flow. IEEE trans. on Image Processing 7 (1998) 359-369 
8. Sethian, J.A.: Level Set Methods and Fast Marching Methods. 2 edn. Cambridge University Press (1999)

9. Farouki, R.T. Curves from motion, motion from curves. In: Curve and Surface Design: Saint- Malo 1999. Vanderbilt Univ. Press (1999)

10. Grady, L.: Random walks for image segmentation. PAMI 28 (2006) 1768-1783

11. Meyer, F., Beucher, S.: Morphological segmentation. Journal of Visual Communication and Image Representation 1 (1990) 21-46

12. Couprie, C., Grady, L., Najman, L., Talbot, H.: Power watersheds: A new image segmentation framework extending graph cuts, random walker and optimal spanning forest. In: ICCV. (2009) $731-738$

13. Li, C., Xu, C., Gui, C., Fox, M.: Distance regularized level set evolution and its application to image segmentation. IEEE Trans. on Image Processing 19 (2010) $3243-3254$

14. Beucher, S., Lantuejoul, C.: Use of watersheds in contour detection. In: International Workshop on Image Processing: Real-time Edge and Motion Detection/ Estimation, Rennes, France. (1979)

15. Cousty, J., Bertrand, G., Najman, L., Couprie, M.: Watershed cuts: Thinnings, shortest path forests, and topological watersheds. PAMI 32 (2010) 925-939

16. Meyer, F.: Topographic distance and watershed lines. Signal Processing 38 (1994) $113-125$

17. Meyer, F.: Inondation par des fluides visqueux. Technical report, Ecole des Mines de Pairs (1993)

18. Vachier, C., Meyer, F.: The viscous watershed transform. Journal of Mathematical Imaging and Vision 22 (2005) 251-267

19. Alahari, K., Kohli, P., Torr, P.: Reduce, reuse and recycle: Efficiently solving multi-label MRFs. In: CVPR. (2008)

20. Kohli, P., Kumar, M., Torr, P.: P3 beyond: Solving energies with higher order cliques. In: CVPR. (2007)

21. Sinop, A.K., Grady, L.: A Seeded Image Segmentation Framework Unifying Graph Cuts And Random Walker Which Yields A New Algorithm. In: ICCV. (2007) 18

22. Allène, C., Audibert, J.Y., Couprie, M., Keriven, R.: Some links between extremum spanning forests, watersheds and min-cuts. Image and Vision Computing 28 (2010) 1460-1471

23. Rother, C., Kolmogorov, V., Blake, A.: Grabcut: interactive foreground extraction using iterated graph cuts. In: SIGGRAPH. (2004) 309-314

24. Martin, D.R., Fowlkes, C.C., Malik, J.: Learning to detect natural image boundaries using local brightness, color, and texture cues. PAMI 26 (2004) 530-549

25. Arbelaez, P., Maire, M., Fowlkes, C., Malik, J.: Contour detection and hierarchical image segmentation. PAMI 33 (2011) 898-916

26. Bertolini, G., Ramat, S.: Identification and recognition of objects in colour stereo images using a hierarchical som. In: Computer and Robot Vision. (2007) 297-304

27. Dailey, D., Cathey, F., Pumrin, S.: An algorithm to estimate mean traffic speed using uncalibrated cameras. IEEE Trans. on Intelligent Transportation Systems 1 (2000) 98-107

28. Omer, O., Tanaka, T.: Region-based weighted-norm approach to video superresolution with adaptive regularisation. In: ICASSP. (2009) 833-836 